\title{
A Platoon-Based Adaptive Signal Control Method with Connected Vehicle Technology
}

\author{
Ning Li, ${ }^{1}$ Shukai Chen, ${ }^{2,3}$ Jianjun Zhu, ${ }^{4}$ and Daniel Jian Sun $\mathbb{D}^{2,3}$ \\ ${ }^{1}$ Ulanqab Vocational College, Ulanqab 012000, Inner Mongolia, China \\ ${ }^{2}$ State Key Laboratory of Ocean Engineering, School of Naval Architecture Ocean and Civil Engineering, \\ Shanghai Jiao Tong University, Shanghai 200240, China \\ ${ }^{3}$ Smart City and Intelligent Transportation (SCIT) Center, Shanghai Jiao Tong University, Shanghai 200240, China \\ ${ }^{4}$ Institute of Computing Technologies, China Academy of Railway Sciences Corporation Limited, Beijing 100081, China \\ Correspondence should be addressed to Daniel Jian Sun; danielsun@sjtu.edu.cn
}

Received 21 November 2019; Accepted 30 January 2020; Published 1 June 2020

Academic Editor: António Dourado

Copyright ( 92020 Ning Li et al. This is an open access article distributed under the Creative Commons Attribution License, which permits unrestricted use, distribution, and reproduction in any medium, provided the original work is properly cited.

One important objective of urban traffic signal control is to reduce individual delay and improve safety for travelers in both private car and public bus transit. To achieve signal control optimization from the perspective of all users, this paper proposes a platoonbased adaptive signal control (PASC) strategy to provide multimodal signal control based on the online connected vehicle (CV) information. By introducing unified phase precedence constraints, PASC strategy is not restricted by fixed cycle length and offsets. A mixed-integer linear programming (MILP) model is proposed to optimize signal timings in a real-time manner, with platoon arrival and discharge dynamics at stop line modeled as constraints. Based on the individual passenger occupancy, the objective function aims at minimizing total personal delay for both buses and automobiles. With the communication between signals, PASC achieves to provide implicit coordination for the signalized arterials. Simulation results by VISSIM microsimulation indicate that PASC model successfully reduces around $40 \%$ bus passenger delay and $10 \%$ automobile delay, respectively, compared with signal timings optimized by SYNCHRO. Results from sensitivity analysis demonstrate that the model performance is not sensitive to the number fluctuation of bus passengers, and the requested CV penetration rate range is around $20 \%$ for the implementation.

\section{Introduction}

Signal light plays a significant role in urban traffic management and control. Adaptive signal control, a state-of-the-art type of traffic control, can remarkably improve the mobility around signalized intersections compared with fixed or actuated controls $[1,2]$. In urban areas, the number of transit buses has boosted in recent years. With proper control strategy favoring the bus and high-occupied vehicles, the controller can effectively reduce passenger delay at intersections. However, recent studies found that the transit signal priority control strategies (TSP) inevitably interrupt automobile traffic flow and increase the control delay for automobile users [3]. To minimize individual traveler delay at intersections, it is necessary to develop a balanced multimodal signal control strategy.

One significant challenge in multimodal adaptive signal control lies in the short-time traffic prediction for cars and transit buses. With the advances in connected vehicle (CV) technology [4], communication between vehicle-infrastructure (V-I) enables reliable traffic information collection. Based on accurate vehicle location and speed data, the generated signal timings may readily match traffic demand fluctuation. Therefore, the CV technology should be incorporated, thus enhancing the multimodal control reliability in traffic management, particularly for signalized intersections.

Diakaki et al. [3] conducted a comprehensive review on recent multimodal control strategies. Some typical adaptive signal control systems, such as SCOOT [5] and SCATS [6], apply a rule-based strategy to grant signal priority to transit buses. Upon receiving a priority request, the controller's reactions involve green extension, red truncation, and special stages. For example, Skabardonis and Geroliminis [7] proposed a strategy to reduce bus control delays and minimize adverse effects on the rest of the traffic. In 
addition, Wadjas and Furth's control strategy [8] aims to assign priority to light rail transits in 3-4 cycles ahead, so that the dwell time became an important factor in arrival estimation. Although the rule-based control strategy is a proactive approach to adjust signal timing for reducing bus delay, it generally deals with only one priority request during the given period. Thus, its application is restricted under oversaturated demand with high bus frequency.

Optimization-based strategies attempt to minimize total disutility (delay, queue length, and stop numbers) by employing nonlinear [9], mix-integer linear [10], or dynamic programming [11]. One advantage of such a control strategy is the nonlimitation on the number of conflicting priorities in the optimization model, compared with rule-based strategies. Christofa et al. [9] developed a model to minimize the total personal delay in mixed traffic lanes based on assumptions that automobile arrival follows a uniform distribution, which is not suitable for closely located intersections. To reduce the potential bus delay at downstream, Ramezani et al. [12] introduced a TSP strategy that comprises of different models to estimate car and bus delays, respectively, for the one-way arterials. However, both strategies assumed an exclusive lane for transit vehicles, indicating that the queues in front of the bus were largely neglected.

To address the disadvantages of the conventional strategies in modelling the mixed traffic conditions, recent research efforts have been dedicated to developing new control methods based on the CV data. He et al. [13] proposed a novel control model called PAMSCOD, which clusters vehicles into platoons to incorporate the arrival patterns subject to upstream intersections. A mixed-integer programming was introduced to solve the problems online based on the platoon or bus serving requests. Hu et al. [14] further exploited the CV technology by adjusting bus speed to ensure the "green wave" under a pair of intersections. Furthermore, Zeng et al. [15] proposed a control strategy named PAPSCCI to model the dynamics of vehicular arrivals. Although vehicles were treated individually to improve the performance, the computational time may be significantly increased under saturated demands.

One common characteristic of the control strategies is the centralized control, which aggregates all signals within one region into a global optimization. Consequently, the increasing number of intersections and the complexity in traffic dynamics often render the problem nonscalable [16]. Therefore, how to achieve progression for multiple traffic modes under a decentralized framework is still a challenging research problem [17].

This study proposes a platoon-based adaptive signal control (PASC) strategy based on the vehicular information extracted from $\mathrm{CV}$ technology. A mixed-integer linear programming (MILP) is developed for online optimization [18]. The main difference between PAMSCOD and the proposed model lies in platoon arrival and delay formulation. In the proposed model, the residual platoon is considered by the constraint so that the delay approximation is simplified. Moreover, the mechanism about implicit coordination is introduced to achieve the progression of neighboring intersections. Under the framework of decentralized control, PASC can save computation time and thus has the potential to be applied in a large urban network. The remainder of the paper is organized as follows. The details of the PASC strategy, as well as the mechanism of implicit coordination between controllers, are proposed in Section 2. In Section 3, a simulation and evaluation platform is developed based on VISSIM. Then, an arterial road, Dongchuan Road, in Minhang District, Shanghai, was modeled to demonstrate model effectiveness. Finally, Section 5 summarizes the conclusions and offers the suggestions for further studies.

\section{Platoon-Based Adaptive Signal Control}

2.1. Platoon Identification. The critical headway is used to identify the approaching and stopping platoons at intersections. Suppose that $h_{0}$ is the critical headway under $100 \%$ CV penetration rate, then under the penetration rate of $R_{p}$, the critical headway is denoted as $h_{p}$ and calculated as in equation (1). If the detected headway of two consecutive connected vehicles is less than $h_{p}$, the vehicles are treated as part of a platoon; otherwise, they are regarded as individual ones:

$$
h_{p}=\frac{h_{0}}{R_{p}} .
$$

At the same time, with penetration rate $R_{p}$, the number of vehicles in the platoon is $n_{p}$, as calculated in the following equation:

$$
n_{p}=\frac{N}{R_{p}},
$$

where $h_{0}$ indicates the critical time headway which is recognized as platoon and $N$ is the detected number of connected vehicles within a platoon. Moreover, the platoon arrival time at stop line is estimated based on the velocity and position of the leading vehicle.

\subsection{Mixed-Integer Linear Programming Model in PASC.} This section introduces a mixed-integer linear programming (MILP) model for multimodal traffic control optimization. The objective is to minimize the total delay for automobile and bus passengers. The set of constraints incorporates NEMA configuration and traffic dynamics upon the stop bar, including serving cycle estimation, platoon arrival, and split. Variables and data notations for the optimization model are listed in Table 1).

2.2.1. Phase Precedence Constraints. The proposed model is based on the standard NEMA 8-phase configuration. Figure 1 presents the phase structure and precedence constraint for a four-leg intersection [10, 19]. Each ring contains four movements, in which conflicting movements are separated by a barrier so that the first movement in one phase group starts only after all movements in the previous group terminate. The 
TABLE 1: Variables and data notations for the proposed optimization model.

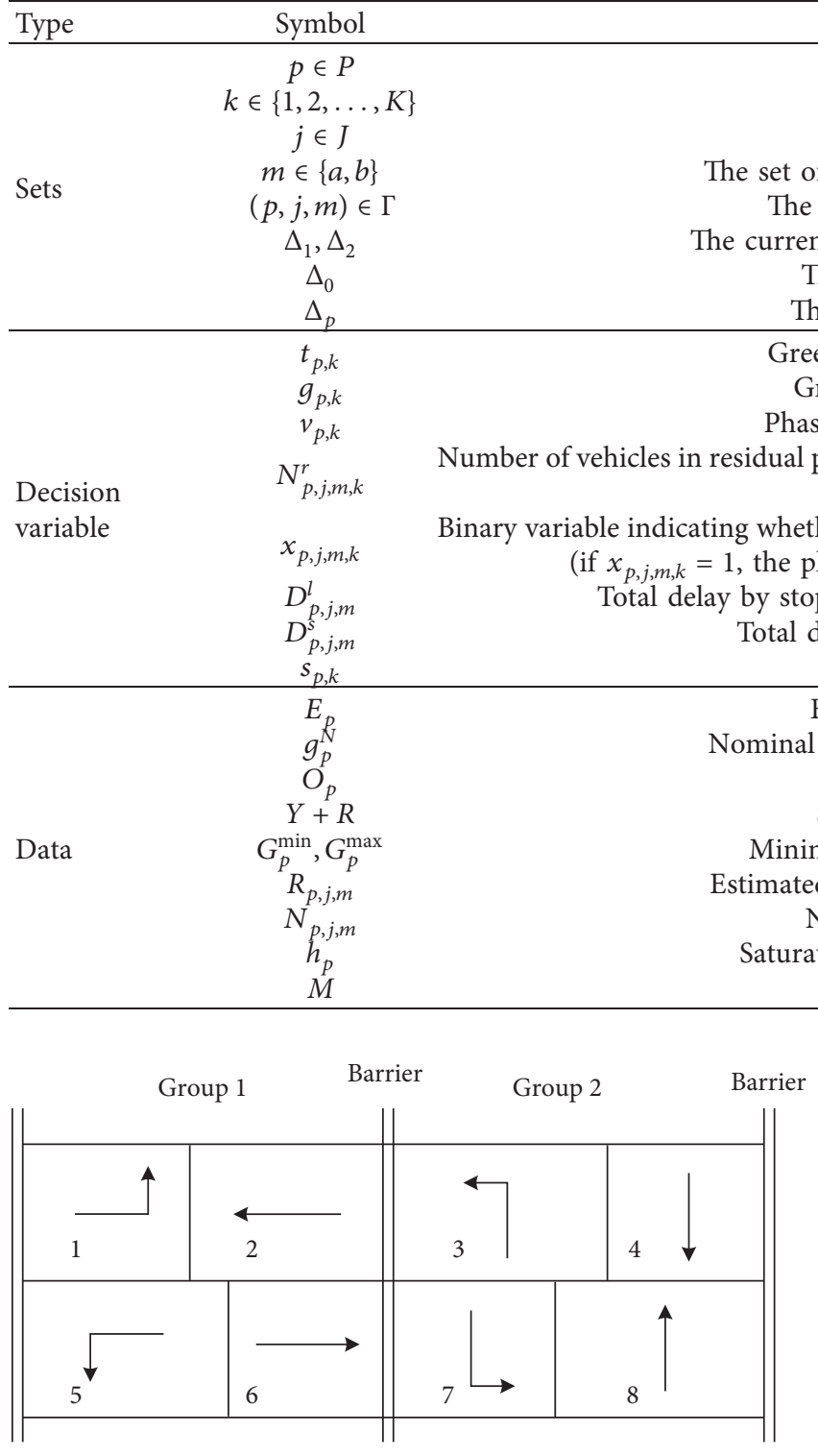

FIGURE 1: NEMA phase with dual-ring structure.

phase precedence constraints are incorporated into the optimization model for each intersection in the control zone.

In this study, the phase precedence constraints (3)-(12) are introduced so that MILP can be formulated at any phase pair. Under NEMA framework, currently active phases belong to following eight phase combinations: 1 and 5, 1 and 6,2 and 5,2 and 6, 3 and 7, 3 and 8, 4 and 7, and 4 and 8 . Thus, NEMA origin phase pair is crucial to calculate the elapsed green time and the minimum green time constraint for current phases. To simplify the model, each intersection is assumed to have the same phase sequence as presented in Figure 1, in which phase pair 1 and 5 is set as the origin phase of a cycle. Then, similar to PAMSCOD [13], the phase precedence constraint is listed as follows:

Definition
The set of phases
The set of cycles
The set of platoons

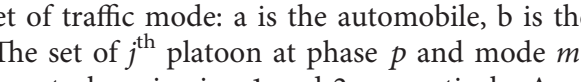

ent phase in ring 1 and 2, respectively, $\Delta_{1}, \Delta_{2} \subset P$

The set of past phases in cycle $1, \Delta_{0} \subset P$

The set of past phases in the current cycle

Green starting time of phase $p$ during cycle $k$

Green duration of phase $p$ during cycle $k$

hase duration time of phase $p$ during cycle $k$

al platoon being cut from platoon $(p, j, m)$ due to shortage of green time in cycle $k\left(N_{p, j, m, k}^{r}>0\right)$

ether platoon $(p, j, m)$ is served before the end of phase $p$ during cycle $k$

stopping the leading vehicle of platoon $(p, j, m), D_{p, j, m}^{l}>0$

tal delay by splitting platoon $(p, j, m), D_{p, j, m}^{s}>0$ Slack time for phase $p$ in cycle $k$

Elapsed green time for phase $p, p \in \Delta_{0}$

green duration time for past phases in cycle 1 Initial starting time for phase $p$

Sum of yellow and red clearance time

Minimum and maximum green time for phase $p$

arrival time at stop line for platoon $(p, j, m)$

Number of vehicles in platoon $(p, j, m)$

turated vehicle headway at stop line for phase $p$ A large constant

$\forall k$,

$$
\begin{aligned}
& t_{6, k}=t_{5, k}+v_{5, k}+s_{5, k}, \\
& t_{7, k}=t_{6, k}+v_{6, k}+s_{6, k}, \\
& t_{8, k}=t_{7, k}+v_{7, k}+s_{7, k},
\end{aligned}
$$

$$
\begin{aligned}
& t_{1, k+1}=t_{4, k}+v_{4, k}+s_{4, k}, \\
& t_{5, k+1}=t_{8, k}+v_{8, k}+s_{8, k},
\end{aligned}
$$

$\forall k$,

$$
\begin{aligned}
& g_{1, k}+g_{2, k}=g_{5, k}+g_{6, k}, \\
& g_{3, k}+g_{4, k}=g_{7, k}+g_{8, k},
\end{aligned}
$$

$\forall k$,

$$
\begin{aligned}
& v_{p, k}=g_{p, k}+Y+R, \quad \forall p, k, \\
& g_{p, 1}=g_{p}^{N}, \quad \forall p \in \Delta_{p},
\end{aligned}
$$




$$
\begin{array}{r}
s_{p, k}=0 \quad \forall p, k \geq 2 \text { or } p \notin \Delta_{1} \cup \Delta_{2}, k=1, \\
G_{p}^{\min } \leq g_{p, k} \leq G_{p}^{\max }, \quad \forall p, k \geq 2 \text { or } p \notin \Delta_{p}, k=1, \\
g_{p, k} \geq \max \left\{E_{p}, G_{p}^{\min }\right\}, \quad \forall p \in \Delta_{1} \cup \Delta_{2}, k=1 .
\end{array}
$$

Constraints (3)-(6) represent the phase sequence for dual-ring phase structure, and the barrier constraint between two phase groups is modeled by constraint (7). It is assumed that each phase starts with clearance time, and consequently, constraint (3) indicates the initial green start time within a cycle. A phase set $\Delta_{p}$ represents the past phases, in which the green time duration is determined by constraint (9). For currently active phases denoted in sets $\Delta_{1}$ and $\Delta_{2}$, the minimum green time constraint is defined as the larger value of the elapsed green time and the minimum green time as shown in constraint (12). Considering that extra time is possible to be required at current phases to satisfy the barrier constraint [13], the slack variable $s_{p, k}$ is defined to relax the maximum green time constraint as constraint (10).

Figure 2 presents the case that the precedence constraint is formulated. The current horizon starts from phases 2 and 6 with elapsed green time E2 and E6, respectively. The dotted line means the green time for past phases, while the solid line represents the green time to be determined. In this study, the planning horizon is assumed to contain at least two complete cycles after the current cycle. Under this phase precedence constraint, the cycle length is flexible to traffic demand fluctuation.

2.2.2. Traffic Dynamics and Delay Evaluation. In the previous research, delay was used as the most common performance index to evaluate the quality of signal timings $[9,15,20]$. This paper classifies the platoon delay into two types: (1) $D_{p, j, m}^{l}$ : total delay by stopping the leading vehicle of platoon $(p, j, m)$, including the time that leading vehicle waits for the end of red time and the departure of front platoons; (2) $D_{p, j, m}^{s}$ : total delay by splitting platoon $(p, j, m)$ if the green duration is not enough to discharge the entire platoon. Before delay evaluation, it is necessary to estimate the cycle that the platoon can cross the intersection.
The serving cycle estimation is based on the platoon arrival time at the stop line and the possible delay by stopping the leading vehicle of platoon. Figure 3 illustrates the two possible cycles serving the platoons around the intersection. For platoon $(p, 1, m)$, it is clear that it will pass the stop line in cycle $k$. Meanwhile, whether the leading vehicle of platoon $(p, 2, m)$ can pass the intersection at cycle $k$ depends on the free-flow speed and the amount of time waiting for the discharge of platoon $(p, 1$, $m$ ). The traffic dynamics are described by the following constraints:

$$
\begin{aligned}
R_{p, j, m}+D_{p, j, m}^{l}-\left(t_{p, k}+g_{p, k}\right) \leq\left(1-x_{p, j, m, k}\right) M, \quad \forall(p, j, m) & \in \Gamma, k \\
\sum_{k} x_{j, p, m, k} & =1, \quad \forall(p, j, m) \in \Gamma,
\end{aligned}
$$

where $R_{p, j, m}+D_{p, j, m}^{l}$ is the actual platoon departure time considering the delay by stopping the leading vehicle of platoon, $t_{p, k}+g_{p, k}$ is the end of green time for phase $p$ in cycle $k$, and $x_{p, j, m, k}$ indicates that whether platoon $(p, j, m)$ departs during cycle $k$.

As depicted in Figure 3, it is figured out that if the leading vehicle of platoon $(p, 2, m)$ arrives at stop line before the end of green time (Case 1), then cycle $k$ is selected as serving cycle. Otherwise, the entire platoon has to wait until the beginning of green time in cycle $k+1$ (Case 2). Constraint (14) ensures that only one cycle in the planning horizon is selected to serve the leading vehicle of the platoon.

For simplicity, the platoon is assumed to travel through the intersection with no dispersion effect. Consequently, all vehicles within one platoon keep the same headway and the vehicle trajectories are parallel all time based on kinematic theory [21]. This means that all vehicles within the platoon experience the same delay, as shown in Figure 4. Therefore, the average delay is denoted as $\left(D_{p, j, m}^{l} / N_{p, j, m}\right)$, which is the difference between the possibly earliest departure time of the leading vehicle and the estimated arrival time at stop line with free-flow speed. To this end, the delay by stopping the leading vehicle in platoon is presented as follows:

$$
\begin{gathered}
\left(\frac{D_{j, p, m}^{l}}{N_{p, j, m}}\right) \geq t_{p, k}+\sum_{m} \sum_{j_{1}=1}^{j_{1}<j} N_{p, j_{1}, m} \cdot \frac{h_{p}}{l_{p}-R_{p, j, m}-\left(1-x_{p, j, m, k}\right) M}, \quad \forall p, k=1 \text { or } p \in \Delta_{p}, k=2, \quad(p, j, m) \in \Gamma, \\
\left(\frac{D_{j, p, m}^{1}}{N_{p, j, m}}\right) \geq t_{p, k}+\sum_{m} \sum_{j_{1}=1}^{j_{1}<j} N_{p, j_{1}, m}^{r} \cdot \frac{h_{p}}{l_{p}-R_{p, j, m}-\left(1-x_{p, j, m, k}\right) M}, \quad \forall p, k>2 \operatorname{or} p \notin \Delta_{0}, k=2, \quad(p, j, m) \in \Gamma,
\end{gathered}
$$

where $t_{p, k}+\sum_{m} \sum_{j_{1}=1}^{j_{1}<j} N_{p, j_{1, m}} \cdot h_{p} / l_{p}$ represents the earliest departure time for the leading vehicle of platoon $(p, j, m)$ in the first cycle length from the moment the MILP is formed, including the phases after $\Delta_{1}$ and $\Delta_{2}$ in current cycle and next cycle's phases belonging to $\Delta_{0}$. For subsequent phases, the residual part of preceding platoons $N_{p, j_{1}, m}^{r}$ should be considered in constraint (16). The earliest departure time is the summation of starting time of phase $p$ and the amount of 


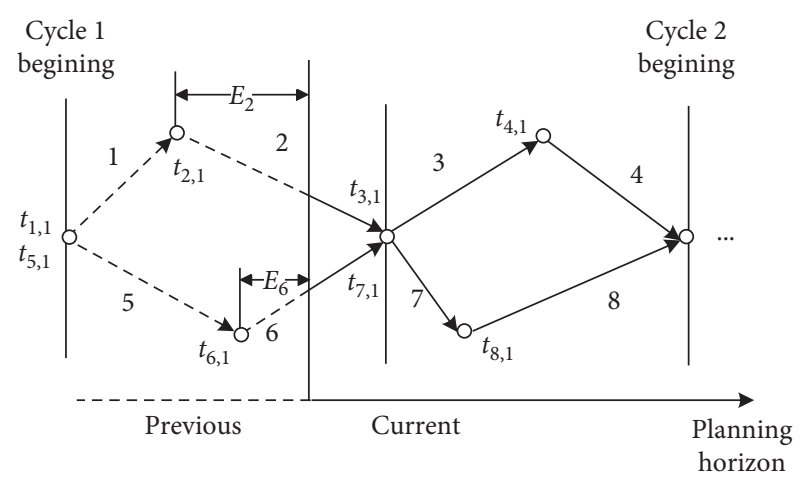

FIgURE 2: Example of planning horizon and current phases.

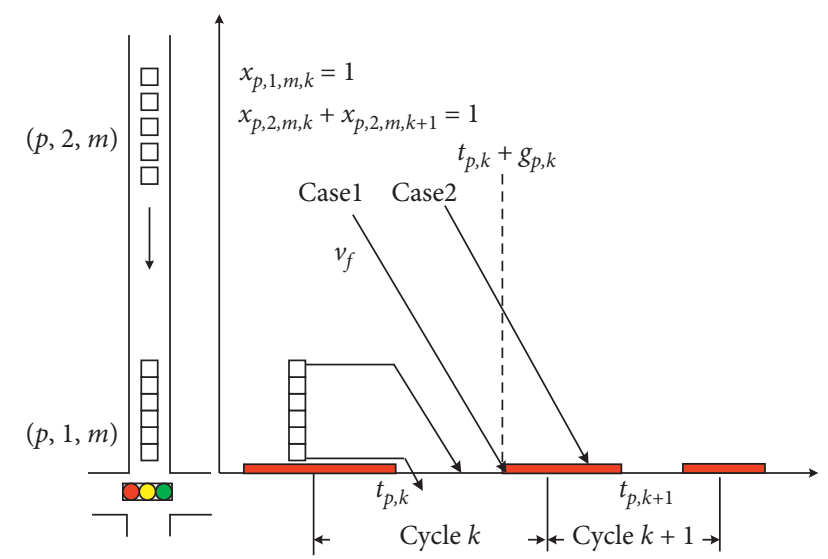

Figure 3: Platoon serving cycle estimation.

time for discharging platoons in front of $(p, j, m)$. The effects of the multiple lanes to saturated headway are considered by introducing the lane number $l_{p}$.

Figure 4 illustrates the calculation for the delay by stopping the leading vehicle delay $D_{p, j, a}^{l}$. Platoon $(p, 2, m)$ is traveling towards the intersection while platoon $(p, 1, m)$ has already been stopped at the stop line. Because platoon $(p, 1, m)$ needs $N_{p, 1, m} \cdot\left(h_{p} / l_{p}\right)$ duration for discharging, platoon $(p, 2, m)$ may consequently join the platoon tail and thus experience delays. It should be noted that since the green duration is enough for discharging all vehicles in platoon $(p, 2, m)$, platoon split does not occur and no vehicles are left behind.

If the assigned green time is not enough to serve all vehicles within a platoon, the platoon will be split into two components, and the remaining part has to experience extra control delay $D_{p, j, a}^{s}$, i.e., red duration between cycle $k$ and cycle $k+1$. Based on the assumption with no dispersion, it is identified that each vehicle within the residual platoon experiences the same delay duration as depicted in Figure 5.

Figure 5 illustrates the generation of delay incurred by splitting the moving platoon. First, the platoon $(p, 2, m)$ is stopped to wait for discharge of the front queues. After the platoon restarts, only a part of vehicles departures from the stop line, while the remaining vehicles become the first platoon for cycle $k+1$. Since the bus is treated as a special

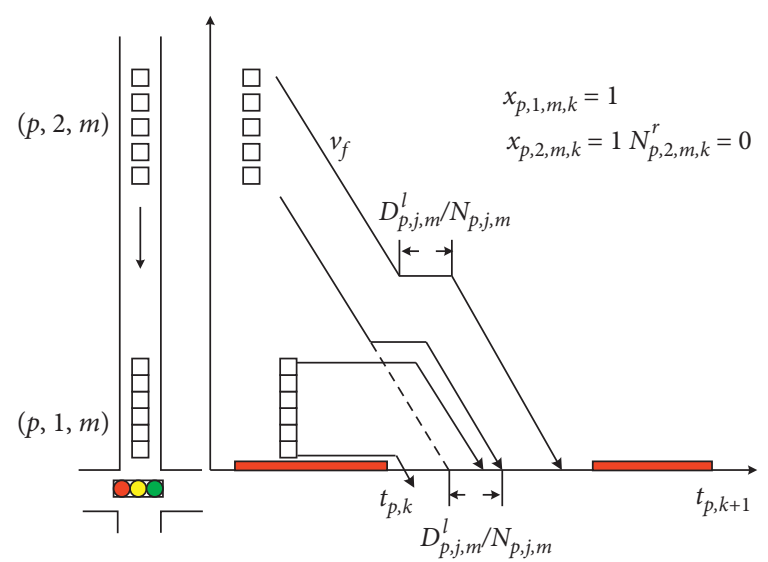

FIGURE 4: Delay incurred by stopping the leading vehicle in platoon $(j=2)$.

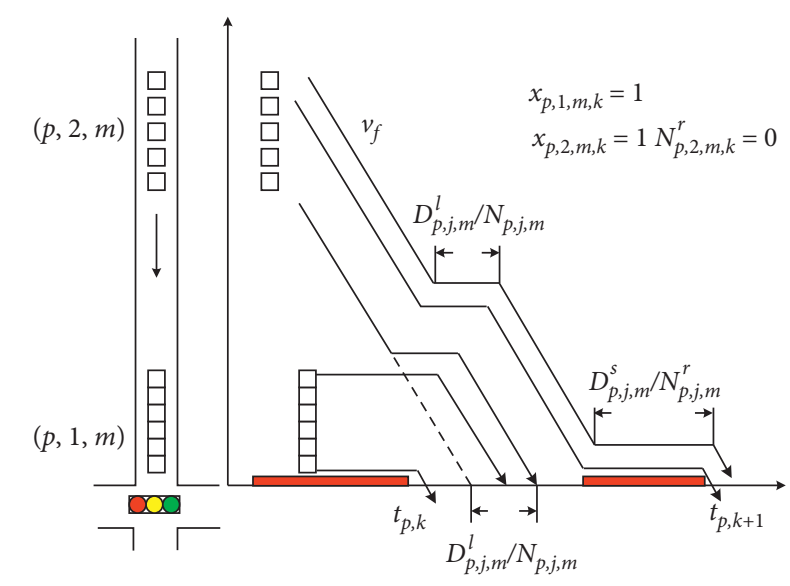

FIGURE 5: Delay incurred by splitting platoon $(j=2)$.

platoon with individual vehicle, the size of residual platoon is not considered. The number of residual platoon before the end of cycle $k$ is then formulated as follows:

$$
\begin{aligned}
N_{p, j, m, k}^{r} \geq & N_{p, j, m}-\frac{t_{p, k}+g_{p, k}-R_{p, j, m}-D_{p, j, m}^{l}}{h_{p} \cdot l_{p}} \\
& -\left(1-x_{p, j, m, k}\right) M, \quad \forall(p, j, m) \in \Gamma, k, m=a,
\end{aligned}
$$

where $t_{p, k}+g_{p, k}-R_{p, j, k}-D_{p, j, m}^{l}$ represents the remaining green time for discharging platoon $(p, j, m)$ when the leading vehicle in the platoon can pass through intersection during cycle $k$. Two situations may occur if the number of remaining vehicles $N_{p, j, m, k}^{r}$ is equal to 0 . The first is that the leading vehicle of platoon $(p, j, m)$ does not arrive at the stop line in cycle $k$, and the second is that the assigned green time allows the entire platoon to depart from the intersection. Both scenarios do not produce the platoon splitting delay.

Given the number of residual vehicles in platoon $(p, j, m)$ before the end of cycle $k$, the total delay incurred by splitting platoon can be presented as follows: 


$$
D_{j, p, m}^{s} \geq N_{j, p, m, k}^{r} \cdot\left(t_{p, k+1}-t_{p, k}-g_{p, k}\right), \quad \forall(p, j, m) \in \Gamma, k,
$$

where $t_{p, k+1}-g_{p, k}-t_{p, k}$ is the effective red duration between cycle $k$ and $k+1$. Unfortunately, this expression makes the constraint nonlinear. Consequently, the delay incurred by splitting platoon is approximated by replacing the component of effective red time with nominal effective red time $[20,22]$. Thus, constraint (18) can be reformulated as follows:

$$
D_{p, j, m}^{s} \geq N_{p, j, m, k}^{r} \cdot\left(C^{N}-g_{p}^{N}\right), \quad \forall(p, j, m) \in \Gamma, k,
$$

where $C^{N}$ represents the nominal cycle length.

2.2.3. Model Formulation. In the proposed model, the objective function is to minimize the total passenger delay for automobile and bus users, which is achieved by weighting delay by vehicular passenger occupancy collected by CV technology. Compared with the vehicle-based delay objective function, the person-based delay objective function can better address conflicting priority for bus services [23]. A summary of model is presented as follows:

$$
\begin{array}{ll}
\text { objective function: } & \sum_{(j, p, m) \in \Gamma} \operatorname{Occ}_{p, j, m} \cdot\left(D_{p, j, m}^{l}+D_{p, j, m}^{s}\right) \\
& \text { phase precedence constraint: (3) - (12) } \\
& \text { serving cycle estimation : (13) and (14) } \\
& \text { delay by stopping leading vehicle: (15)and (16) } \\
& \text { number of residual queue : (17) } \\
\text { s.t. } & \text { delay by splitting platoon: (19), }
\end{array}
$$

where $\mathrm{Occ}_{p, j, m}$ is the passenger occupancy for platoon $(p, j, m)$, and the controlled variables are binary decision variables, and all other variables are nonnegative.

As discussed, the proposed control model aims to serve all identified platoons in three cycles under the framework of rolling horizon. The optimization model is formed and solved every $30 \mathrm{~s}$ to implement the signal control. For simplicity, only two significant platoons were considered for noncoordinated phases, while at least one platoon at upstream intersection is estimated for the coordinated phases (phases 2 and 6). Since the queues at upstream stop bar will approach to the current intersection as one or more platoons, the size and starting time of those platoons could be roughly estimated by the location of upstream vehicles and the signal timing plans [13]. Location data on the upstream vehicle are collected by CV technology, while the signal timing is extracted through communication between signals.

2.3. Communication between Signals. For a signalized arterial, the proposed control model optimizes signal timing for each intersection in a fixed sequence. The implicit coordination depends on optimized signal timings of individual controller and the way of unknown signal timing estimation. In this study, the latest historical signal timings are used to predict upstream phase duration [17].
For the coordinated phases at upstream intersection, phase durations can be obtained from the last optimization results. For the signal timings out of previous rolling horizon, the latest historical green time is assigned. The unknown green time duration for phase $p$ in cycle $k$ is then calculated as follows:

$$
\widehat{g}_{p, k}=g_{p, k-1}, \quad \forall p, k,
$$

where $\widehat{g}_{p, k}$ is the estimated green time for upstream intersection and $g_{p, k-1}$ is the actual realized green time on cycle $k-1$.

Based on the phase precedence constraints presented in Section 2.1, the estimated starting time of coordinated phase is obtained at upstream intersections. Therefore, the upstream platoon arrival time at current intersection can be calculated as follows:

$$
R_{p, j, m}=\widehat{t}_{p, k}+t_{d}, \quad \forall(p, j, m) \in_{d}, k
$$

where $\widehat{t}_{p, k}$ is the estimated starting time for phase $p$ at upstream intersection, $t_{d}$ is the platoon travel time from upstream intersection to the current stop line, and $\Gamma_{d}$ is the set of platoons which have not passed the upstream intersection. With the estimated arrival time of platoon discharged from upstream intersection as input, implicit coordination between neighboring intersections is fulfilled.

\section{Simulation Case Study}

To evaluate the effectiveness of the proposed model, a simulation and evaluation platform was developed in $\mathrm{C}++$ language. The system contains VISSIM with COM (component object module) as a simulation module and IBM/CPLEX solver serving for optimization purpose. For every 30 seconds, vehicular information was extracted for the optimization, and CPLEX was used to find the optimal solution, so that the generated signal timings can be implemented into the VISSIM simulations [24].

3.1. Simulation Test Bed. Simulation tests were conducted based on the arterial segment of Dongchuan Road (D.C. Road), ranging from Cangyuan Road (C.Y. Road) to Anning Road (A.N. Road). As shown in Figure 6, three conflicting bus routes (routes 4,11, and 16) are operated in the network, in which one route travels on the Dongchuan Road and the others travel on the cross streets. All far-side bus stops are located near the exit of upstream intersection. In the arterial network, D.C. Road/C.Y. Road and D.C. Road/A.N. Road are entry intersections of control zones, and vehicles arrive randomly at one coordinated phase (phase 2 or 6). In D.C. Road/H.M. Road, vehicles generally arrive in platoons at both coordinated phases subject to upstream signal controls [25].

Table 2 presents three demand scenarios to validate the proposed control model. The level of saturation is measured by Intersection Capacity Utilization (ICU) in SYNCHRO. It is assumed that all buses were equipped with CV technology, so that the information on bus location and speed is 


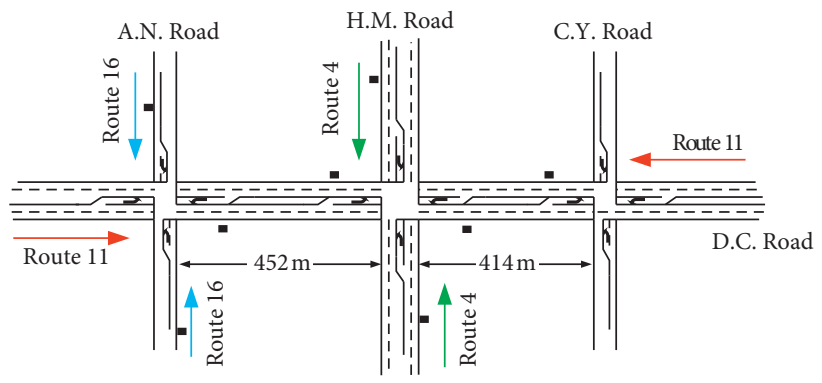

- Bus stop

Figure 6: Network layout and bus routes.

TABle 2: Demand scenario (veh/h).

\begin{tabular}{|c|c|c|c|c|c|c|c|c|c|c|}
\hline \multirow{2}{*}{\multicolumn{2}{|c|}{ Movement }} & \multicolumn{3}{|c|}{$\mathrm{ICU}=0.5$} & \multicolumn{3}{|c|}{$\mathrm{ICU}=0.7$} & \multicolumn{3}{|c|}{$\mathrm{ICU}=0.9$} \\
\hline & & D.C./C.Y & D.C./H.M & D.C./A.N. & D.C./C.Y & D.C./H.M. & D.C./A.N. & D.C./C.Y & D.C./H.M. & D.C./A.N. \\
\hline \multirow{3}{*}{ WB } & $\mathrm{L}$ & 52 & 131 & 50 & 69 & 225 & 122 & 154 & 200 & 190 \\
\hline & Th & 431 & 502 & 515 & 835 & 852 & 659 & 1003 & 1008 & 1025 \\
\hline & $\mathrm{R}$ & 88 & 42 & 113 & 123 & 122 & 86 & 128 & 79 & 109 \\
\hline \multirow{3}{*}{ EB } & $\mathrm{L}$ & 172 & 134 & 147 & 190 & 192 & 227 & 222 & 162 & 217 \\
\hline & Th & 433 & 555 & 519 & 823 & 805 & 842 & 1138 & 1246 & 1362 \\
\hline & $\mathrm{R}$ & 99 & 36 & 50 & 105 & 218 & 117 & 123 & 183 & 94 \\
\hline \multirow{3}{*}{ SB } & $\mathrm{L}$ & 134 & 192 & 59 & 196 & 206 & 78 & 170 & 350 & 64 \\
\hline & $\mathrm{Th}$ & 93 & 259 & 123 & 154 & 447 & 163 & 163 & 542 & 163 \\
\hline & $\mathrm{R}$ & 20 & 39 & 85 & 107 & 98 & 223 & 107 & 92 & 228 \\
\hline \multirow{3}{*}{ NB } & $\mathrm{L}$ & 68 & 90 & 128 & 96 & 144 & 227 & 116 & 190 & 234 \\
\hline & $\mathrm{Th}$ & 66 & 190 & 165 & 154 & 402 & 106 & 156 & 510 & 139 \\
\hline & $\mathrm{R}$ & 75 & 103 & 71 & 138 & 125 & 80 & 186 & 149 & 98 \\
\hline
\end{tabular}

available. During the simulation tests, each bus sends priority requests before arriving at an intersection. The bus occupancy in this study changes from 20 to 30 and then to 40 , under different demand scenarios, which may be based on the average number of passengers collected from APC (Automatic Passenger Counter) devices during the field implementation.

The background signal timing information was obtained from SYNCHRO, including cycle length, phase splits, and offsets. Fixed signal timings were served as the baseline for the proposed model. For each scenario, the simulation test runs with 10 different random seeds based on one-hour duration, including $10 \mathrm{~min}$ warm up period [26-28].

\section{Results and Discussion}

Under the assumption of $100 \%$ penetration rate and average 40 bus passenger occupancy, the simulation test results with three different control strategies are listed in Table 3, namely, the fixed signal timings from SYNCHRO and the proposed control model with person-based and vehicle-based objective functions. Person-based objective function considers passenger occupancy in each bus, while vehicle-based one treats bus with the same weight with cars. Personal delay was chosen as the measurement performance of control strategy for automobiles, bus, and all vehicles. Figures 7 and 8 depict the percentage of change in personal delay from SYNCHRO to the proposed models, with findings summarized as follows.
As shown in Figure 7, the person-based PASC has obvious advantages compared with SYNCHRO, personal delay for each category of vehicle decreases with the rise of traffic demand. Specifically, under low traffic demand ( ICU $=0.5$ ), although the reduction of automobile passenger delay is not significant, the bus passengers experience nearly less $40 \%$ personal delay. More importantly, the decreasing trend of personal delay of all vehicles (more than 20\%) shows that giving signal priority to bus passenger will not sacrifice the benefits of all automobile users. As a result, the person-based PASC actually reduces the delay of all the passengers.

Figure 8 presents the comparison between two PASC strategies with person-based and vehicle-based objective functions. First, the person-based PASC generated more personal delay for automobile passengers by $5 \%-10 \%$ compared with vehicle-based PASC, which is acceptable, since in transit priority, the automobile platoon may wait for longer time to give way to buses in other phases. With the increase of traffic demand, the disruption effect becomes more obvious. However, the person-based PASC still produce less personal delay for all vehicles in the network, again proving its ability to balance the passing priority of all users.

To evaluate the impact of the fluctuation of bus passenger number to the controller's performance, three levels of the bus passenger occupancy (BPO), were set as 20,30, and 40 passengers per bus, respectively. Within the heavy traffic demand $(\mathrm{ICU}=0.9)$, the test results are presented in Figure 9. It can be figured out that the change of BPO has a limited impact on the optimization results. Personal delay of 
TABLE 3: Control performance under different methods (personal delay, seconds).

\begin{tabular}{lcccc}
\hline ICU & Vehicle type & SYNCHRO & PASC (person-based) & PASC (vehicle-based) \\
\hline \multirow{3}{*}{0.5} & Automobile & 24.2 & 23.9 & 22.7 \\
& Bus & 34.7 & 21.3 & 31.6 \\
0.7 & All vehicles & 29.8 & 22.6 & 27.4 \\
& Automobile & 28.5 & 26.2 & 25.0 \\
& Bus & 40.6 & 25.0 & 34.1 \\
0.9 & All vehicles & 33.7 & 25.7 & 29.0 \\
& Automobile & 35.5 & 31.1 & 28.2 \\
& Bus & 51.8 & 29.6 & 38.3 \\
\hline
\end{tabular}

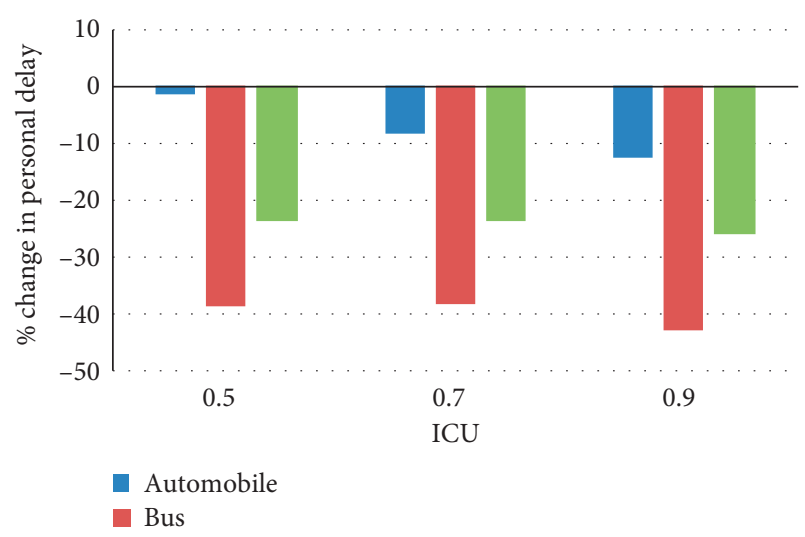

FIGURE 7: Percentage of change in person delay from SYNCHRO to person-based PASC under different traffic demands.

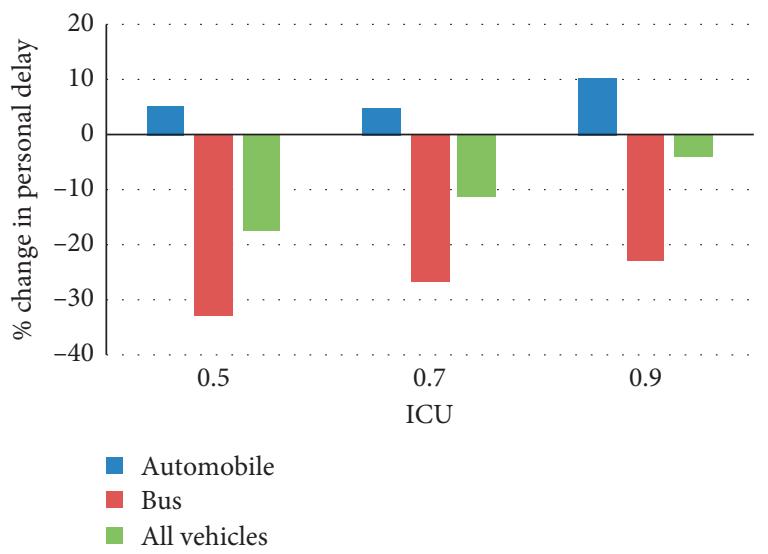

FIgURE 8: Percentage of change in person delay from vehicle-based to person-based PASC under different traffic demands.

automobile passengers keeps almost unchanged, while the personal delay of bus passenger declines slightly with the increase of BPO. As a result, the overall road users would reduce personal delay by $20 \%$.

Since vehicle information collected by CV technology serves as an important input for platoon identification, the market penetration rate of $\mathrm{CV}$ devices largely influences the quality of signal timing. To test the model sensitivity to the penetration rate, the ideal assumption that all vehicles are

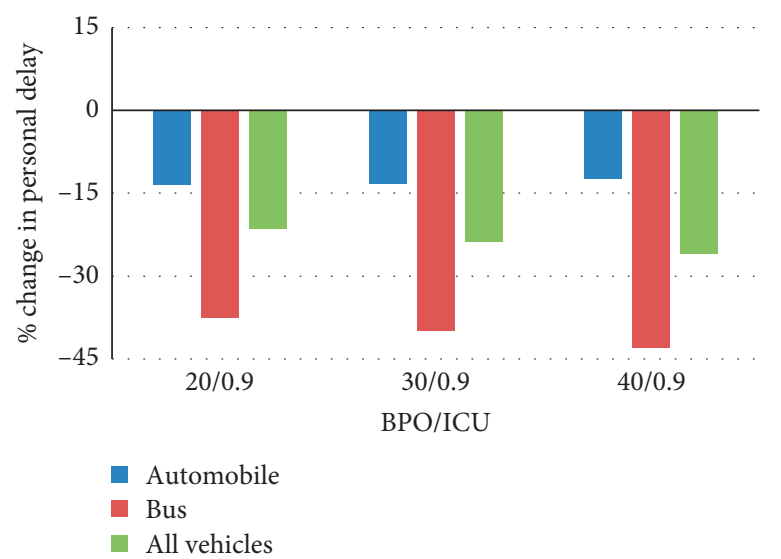

FIgURE 9: Percentage of change in person delay from SYNCHRO to person-based PASC with different BPOs.

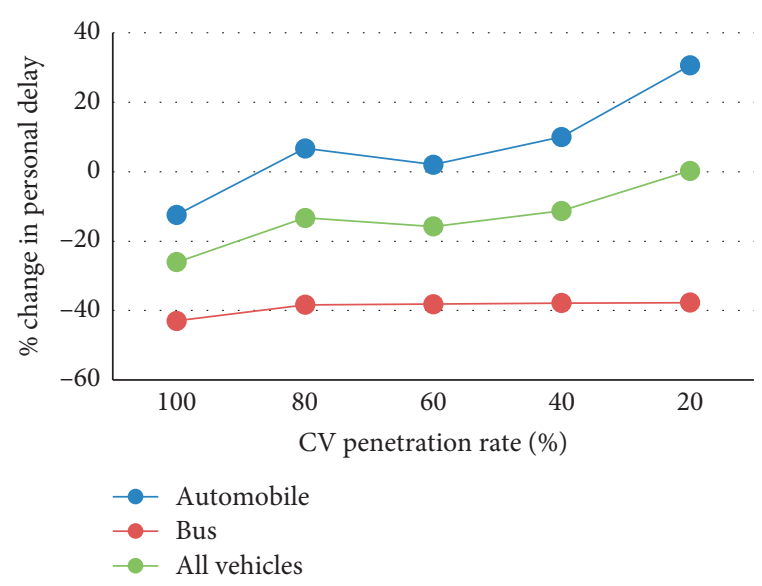

FIGURE 10: Percentage of change in person delay from SYNCHRO to person-based PASC with different $\mathrm{CV}$ penetration rates.

equipped with CV devices was relaxed. Based on the penetration rate ranging from $20 \%$ to $100 \%$ with a $20 \%$ interval, simulation tests were conducted under high demand scenarios $(\mathrm{ICU}=0.9 ; \mathrm{BPO}=40)$. Because all buses are generally equipped with GPS devices, only automobiles are assumed to subject to the penetration rate change. Figure 10 illustrates the percentage of changes in person delay from SYNCHRO to person-based PASC under different penetration rates. 
It can be figured out that personal delay of all types of vehicles increases as fewer vehicles are detected on the road. If the penetration rate is lower than $80 \%$, PASC is likely to generate more personal delay to automobile passengers, and the increasing trend grows obviously under a lower penetration rate. This is probably caused by the estimation error in determining the position of the leading vehicle in the platoon, which can be solved by a more complicated platoon identification algorithm [13]. On the other hand, personal delay of the bus passenger rises slightly, which indicates that the estimation error of the front platoons does not influence the benefits of bus passengers. Considering PASC still reduces personal delay for passengers of all vehicles with more than $20 \%$ penetration rate, it is recommended that the obligatory $\mathrm{CV}$ penetration rate should be set at least as $20 \%$.

\section{Conclusions}

This paper proposed an improved platoon-based adaptive control strategy to provide multimodal traffic management for signalized intersections, assuming that the connected vehicle information is available online. By introducing unified phase precedence constraints, PASC was not restricted by fixed cycle length and offset. A MILP optimization model was developed, in which platoon arrival and discharge dynamics were modeled for delay evaluation. With the communication between controllers, PASC provided implicit signal coordination of neighboring intersections for automobiles and buses.

A simulation and evaluation platform was developed to validate the proposed control strategy. The results indicated that person-based PASC successfully strikes a balance between automobile and bus passengers. At high demand scenario, person-based PASC reduces up to $40 \%$ bus passenger delay and $10 \%$ automobile passenger delay in comparison with SYNCHRO. Although the control performance of person-based PASC for automobile passengers is slightly inferior to vehicle-based PASC, it still reduces all personal delay by around $10 \%$. Through sensitivity evaluation, it was found that the control performance keeps stable with the fluctuation of bus passenger number, and the minimal CV penetration rate is around $20 \%$.

While the results are promising, limitations still exist within the proposed model, especially when the penetration rate is low. Therefore, how to improve the model robustness under a low penetration environment is a crucial question to be explored. Furthermore, additional progression mechanism and/or big data analytics should be incorporated into PASC to improve control performance in a large network $[29,30]$. In future, PASC may be applied to a grid network to evaluate the effectiveness of implicit coordination.

\section{Data Availability}

The data used to support the findings of the study are available from the corresponding author upon request.

\section{Disclosure}

The previous version of the manuscript was presented in Transportation Research Board $95^{\text {th }}$ Annual Meeting. Any opinions, findings, and conclusions or recommendations expressed in this paper are those of the authors and do not necessarily reflect the views of the sponsors.

\section{Conflicts of Interest}

The authors declare that they have no conflicts of interest.

\section{Acknowledgments}

The research was funded in part by the National Natural Science Foundation of China (71971138), the Shanghai Municipal Natural Science Foundation (17ZR1445500), China, and the Shanghai "Science and Technology Innovation Action Plan"- "One Belt and One Road" International Cooperation Project (19210745600).

\section{References}

[1] R. Haijema and E. M. T. Hendrix, "Traffic responsive control of intersections with predicted arrival times: a Markovian approach," Computer-Aided Civil and Infrastructure Engineering, vol. 29, no. 2, pp. 123-139, 2014.

[2] J. Sun and L. Zhang, "Vehicle actuation based short-term traffic flow prediction model for signalized intersections," Journal of Central South University, vol. 19, no. 1, pp. 287-298, 2012.

[3] C. Diakaki, M. Papageorgiou, V. Dinopoulou, I. Papamichail, and M. Garyfalia, "State-of-the-art and practice review of public transport priority strategies," IET Intelligent Transport Systems, vol. 9, no. 4, pp. 391-406, 2014.

[4] S. I. Guler, M. Menendez, and L. Meier, "Using connected vehicle technology to improve the efficiency of intersections," Transportation Research Part C: Emerging Technology, vol. 46, pp. 121-131, 2014.

[5] F. M. Oliveira-Neto, C. F. G. Loureiro, and L.-D. Han, “Active and passive bus priority strategies in mixed traffic arterials controlled by SCOOT adaptive signal system: assessment of performance in Fortaleza," Journal of the Transportation Research Board, vol. 2128, pp. 20-29, 2009.

[6] P. R. Lowrie, "SCATS: the Sydney coordinated adaptive traffic system-principles, methodology, algorithms," in Proceedings of the IEEE International Conference on Road Traffic Signaling, pp. 67-70, London, UK, 1982.

[7] A. Skabardonis and N. Geroliminis, "Real-time monitoring and control on signalized arterials," Journal of Intelligent Transportation Systems, vol. 12, no. 2, pp. 64-74, 2008.

[8] Y. Wadjas and P. G. Furth, "Transit signal priority along arterials using advanced detection," Transportation Research Record: Journal of the Transportation Research Board, vol. 1856, no. 1, pp. 220-230, 2003.

[9] E. Christofa, I. Papamichail, and A. Skabardonis, "Personbased traffic responsive signal control optimization," IEEE Transactions on Intelligent Transportation Systems, vol. 14, no. 3, pp. 1278-1289, 2013.

[10] L. Head, D. Gettman, and Z. Wei, "Decision model for priority control of traffic signals," Transportation Research Record: Journal of the Transportation Research Board, vol. 1978, no. 1, pp. 169-177, 2006. 
[11] S. Sen and K. L. Head, "Controlled optimization of Phases at an intersection," Transportation Science, vol. 31, no. 1, pp. 5-17, 1997.

[12] M. Ramezani, R. Burgener, and N. Geroliminis, "Optimization of traffic signals for transit priority in arterials with dedicated bus lanes and stochastic arrivals: a system-oriented approach," in Proceedings of the 94th Annual Meeting of the Transportation Research Board, Washington, DC, USA, 2015.

[13] Q. He, K. L. Head, and J. Ding, "PAMSCOD: platoon-based arterial multi-modal signal control with online data," Transportation Research Part C: Emerging Technologies, vol. 20, no. 1, pp. 164-184, 2012.

[14] J. Hu, B. B. Park, and Y.-J. Lee, "Coordinated transit signal priority supporting transit progression under connected vehicle technology," Transportation Research Part C: Emerging Technologies, vol. 55, pp. 393-408, 2015.

[15] X. Zeng, X. Sun, Y. Zhang, and L. Quadrifoglio, "Person-based adaptive priority signal control with connected-vehicle information," Transportation Research Record: Journal of the Transportation Research Board, vol. 2487, no. 1, pp. 78-87, 2015.

[16] M. Mehrabipour and A. Hajbabaie, "A cell-based distributedcoordinated approach for network-level signal timing optimization," Computer-Aided Civil and Infrastructure Engineering, vol. 32, no. 7, pp. 599-616, 2017.

[17] J. Li and H. M. Zhang, "Coupled linear programming approach for decentralized control of urban traffic," Transportation Research Record: Journal of the Transportation Research Board, vol. 2439, no. 1, pp. 83-93, 2014.

[18] J. Zhao, Y. Liu, and T. Wang, "Increasing signalized intersection capacity with unconventional use of special width approach lanes," Computer-Aided Civil and Infrastructure Engineering, vol. 31, no. 10, pp. 794-810, 2016.

[19] L. Head, D. Gettman, D. M. Bullock, and T. Urbanik, "Modeling traffic signal operations with precedence graphs," Transportation Research Record: Journal of the Transportation Research Board, vol. 2035, no. 1, pp. 10-18, 2007.

[20] E. Christofa, K. Ampountolas, and A. Skabardonis, "Arterial traffic signal optimization: a person-based approach," Transportation Research Part C: Emerging Technologies, vol. 66, pp. 27-47, 2015.

[21] M. Lighthill and G. Whitham, "On kinematic waves. II. A theory of traffic flow on long crowded roads," Proceedings of the Royal Society of London. Series A. Mathematical and Physical Sciences, vol. 229, no. 1178, pp. 317-345, 1955.

[22] Q. He, K. L. Head, and J. Ding, "Multi-modal traffic signal control with priority, signal actuation and coordination," Transportation Research Part C: Emerging Technologies, vol. 46, pp. 65-82, 2014.

[23] S. Chen and D. J. Sun, "An improved adaptive signal control method for isolated signalized intersection based on dynamic programming," IEEE Intelligent Transportation Systems Magazine, vol. 8, no. 4, pp. 4-14, 2016.

[24] D. J. Sun, L. Zhang, and F. Chen, "Comparative study on simulation performances of CORSIM and VISSIM for urban street network," Simulation Modelling Practice and Theory, vol. 37, pp. 18-29, 2013.

[25] D. J. Sun, Y. Zhang, R. Xue, and Y. Zhang, "Modeling carbon emissions from urban traffic system using mobile monitoring," Science of the Total Environment, vol. 599-600, pp. 944-951, 2017.

[26] D. J. Sun and L. Elefteriadou, "A driver behavior-based lanechanging model for urban arterial streets," Transportation Science, vol. 48, no. 2, pp. 184-205, 2014.
[27] D. J. Sun and L. Elefteriadou, "Lane-changing behavior on urban streets: an "in-vehicle" field experiment-based study," Computer-Aided Civil and Infrastructure Engineering, vol. 27, no. 7, pp. 525-542, 2012.

[28] D. J. Sun and L. Elefteriadou, "Research and implementation of lane-changing model based on driver behavior," Transportation Research Record: Journal of the Transportation Research Board, vol. 2161, no. 1, pp. 1-10, 2010.

[29] D. J. Sun and X. Ding, "Spatiotemporal evolution of ridesourcing markets under the new restriction policy: a case study in Shanghai," Transportation Research Part A: Policy and Practice, vol. 130, pp. 227-239, 2019.

[30] D. J. Sun, K. Zhang, and S. Shen, “Analyzing spatiotemporal traffic line source emissions based on massive didi online carhailing service data," Transportation Research Part D: Transport and Environment, vol. 62, pp. 699-714, 2018. 